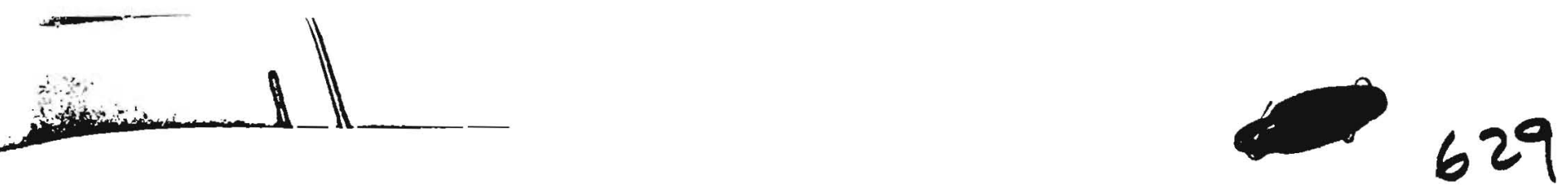

\title{
Liver Transplantation for Fulminant Hepatic Failure
}

\author{
SHUNZABURO IWATSUKI, M.D., CARLOS O. ESQUIVEL, M.D., Ph.D., \\ ROBERT D. GORDON, M.D., BYERS W. SHAW Jr., M.D., \\ THOMAS E. STARZL, M.D., Ph.D., ROBERT R. SHADE, M.D., \\ and DAVID H. VAN THIEL, M.D.
}

Fulminant hepatic failure is the clinical syndrome associated with acute massive necrosis of liver cells. It is characterized by the sudden onset of progressive jaundice, decrease in size of the liver, fetor hepaticus, and hepatic encephalopathy within eight weeks of acute illness. This syndrome carries a mortality rate of more than $80 \%$ when the patient develops grand IV hepatic encephalopathy. ${ }^{1,2}$ The depth of encephalopathy is important as an index of the severity of hepatic failure and in the assessment of the prognosis. Other systemic manifestations of fulminant hepatic failure include hypoglycemia, metabolic acidosis, respiratory failure, renal impairment, coagulopathy, and bacteremia.

Fulminant hepatic failure is treated with nonspecific supportive medical measures. Plasma or whole blood exchange and charcoal hemoperfusion therapy have been tried with some success. ${ }^{3,4}$ These treatments may prevent or correct some of the systemic complications of this syndrome and allow enough time for the liver to regenerate. However, when the liver cells are destroyed beyond the level of potential regeneration, all supportive measures are fruitless without liver replacement. For many years, there has been an interest in treating fulminant hepatic failure by liver transplantation. Until recently, this could not be achieved because of a generally low success rate (one-year survival rate of $33 \%$ with azathioprine-prednisone therapy) and a lack of organ donors at the appropriate time. Both

From the Departments of Surgery and Medicine, University of Pittsburgh Health Center, University of Pittsburgh, Pittsburgh, Pennsylvania.

Reprint requests: Dr. Iwatsuki, Dept. of Surgery, 3601 Fifth Ave., Room 103, Falk Clinic, Pittsburgh, PA 15213. of these obstacles had been partly eliminated in the last few years. The one-year survival after liver transplantation has improved to over $70 \%$ with cyclosporine-steroid therapy, and more donors have become available due to an increasing public aware. ness of the need.

When a complete summary of a 19-year experience was brought up-to-date to May $1982,{ }^{5}$ there had been only one attempt at the treatment of fulminant hepatic failure by transplantation, the recipient being a youth with fulminant B-virus hepatitis (OT 87). Numerous other candidates had been referred either to the University of Colorado (through 1980) or to the University of Pittsburgh. However, almost all of these patients died while a search was being conducted for an appropriate donor. A very few recovered. During the last three years, durther attempts have been made to treat patients with fulminant hepatic failure, and now with some success.

We report here eight attempts at hepatic replacement under these dire circumstances during the last 36 months. The causes of massive hepatic necrosis were variable, ranging from drug intoxication to viral hepatitis. Although the perioperative mortality has been high, primarily because of irreversible brain injury and severe systemic complications, four of these eight patients have recovered fully.

In this article, we will consider three issues. First will be the appropriate bare bones evaluation and preoperative management that are designed to allow a decision to be made about the potential reversibility of the acute hepatic disease. The second will consider the special technical and other perioperative problems that may be important. Third, we will report our results. 


\section{PRETRANSPLANT EVALUATION AND MANAGEMENT}

Acute massive hepatic necrosis usually results from viral hepatitis (Type A, Type B, or a presumed non-A, non-B virus infection), drug (hypersensitivity or overdose), or toxic chemicals. The cause of fulminant hepatic failure must be determined not only to assess the prognosis of the acute illness, but also to prevent further injury to an already damaged liver. A carefully obtained medical history usually will disclose the exposure to a hepatitis virus or to toxic chemicals, recently administered potentially hepatotoxic medications, and the accidental or suicidal overdose of drugs. Serologic tests for hepatitis viruses and chemical analysis of blood for toxic agents can often confirm a suspected etiology.

Determination of the prognosis of the acute illness is a prime objective of the workup of patients with massive hepatic necrosis who are being considered for liver transplantation. Development of hepatic encephalopathy and its rapid progression is the most ominous prognostic sign, and papillary edema indicates significant cerebral edema which leads to brain death. After grade III or IV encephalopathy develops, systemic complications such as renal failure, gastrointestinal hemorrhage, bacteremia, or spontaneous bacterial peritonitis become increasingly frequent. These are also grave prognostic signs. Since liver transplantation in the presence of grade IV encephalopathy or in the presence of severe systemic complications is usually unsuccessful, the timing of the liver replacement before these complications are themselves irreversible is essential. Rapidly reducing size of the liver, increasing serum bilirubin, markedly prolonged prothrombin time, hypoglycemia, and progressive renal failure (hepatorenal syndrome) are all indications of serious hepatic failure which may require liver replacement. A decision for liver transplantation for fulminant liver failure is one of the most difficult clinical judgments even for an experienced hepatologist who has followed the patient on an hourly basis since the onset of illness. As the results of liver transplantation have improved significantly over the last five years, the decision seems to have become less agonizing than before.

As soon as potential need of liver transplantation is raised for a patient with fulminant hepatic failure, the transplant team should begin a search for a suitable organ donor. Age, sex, ABO-blood group, weight, and height of the potential recipient are reported to the regional organ bank, and these also are listed on a nationwide computer system. At the same time, anatomic and physiologic conditions of the potential recipient are assessed. Past medical histories are carefully reviewed, with particular attention to previous abdominal surgery and past cardiovascular and pulmonary diseases. The size of the liver, state of the biliary system, and patency of the portal vein are determined with ultrasonography. Infectious complications such as pneumonia, urinary tract infection, bacteremia or spontaneous bacterial peritonitis are looked for and if present, must be treated adequately before operation. The hemodynamic status of the patient should be monitored closely by using a Swan-Ganz catheter. Intravenous fluid therapy is managed carefully, particularly when renal impairment is developing. Endotracheal intubation and respiratory assist is necessary to prevent the development of aspiration pneumonia or to correct acid-based imbalances. Gastrointestinal bleeding is a common complication of fulminant hepatic failure. Gastric acidity should be reduced by intensive antiacid therapy, and coagulation abnormalities must be corrected by fresh frozen plasma, cryoprecipitated plasma, platelet transfusion, and/or epsilon-aminocaproic acid. In order to prevent the patient with grade IV encephalopathy from progressing to brain death from cerebral edema, intracranial pressure may be reduced by mannitol infusion, or the encephalopathy may be reduced by plasma exchange or charcoal hemoperfusion, if indicated. Aggressive medical management to prevent or treat systemic complications of fulminant hepatic failure plays an important role in the successful therapy of this grave illness, whether or not liver replacement is decided upon.

\section{INTRAOPERATIVE MANAGEMENT}

The hemodynamic changes in fulminant hepatic failure are similar to those in septic shock, an increase in cardiac output and a decrease in systemic vascular resistance. Further hemodynamic derangements during transplant operation can lead to cardiac failure or arrest, which can aggravate the damage to an already injured central nervous system. Irreversible cerebral cortical damage or even brain death has been all too common when liver replacement was carried out on a patient in grade IV encephalopathy. The most significant hemodynamic changes during liver transplantation occur when the recipient's diseased liver is being removed and replaced by the donor liver (anhepatic phase). In the past, both the inferior vena caval flow and the portal venous flow were interrupted during this 
anhepatic phase, which added significant mortality and morbidity to the operation. The new venovenous bypass system without systemic heparinization ${ }^{6}$ has virtually eliminated this period of hemodynamic instability. The inferior caval and portal venous blood are returned to the superior vena cava system by the veno-venous bypass system during the anhepatic phase. The cardiac output is thereby maintained. This bypass system should be used in every liver transplantation for fulminant hepatic failure because even a slight further decrease in cerebral blood flow can result in irreversible brain injury. Perioperative electroencephalographic (EEG) monitoring is useful to diagnose additional cerebral insults and to correct them, if possible, with mannitol infusion to reduce intracranial pressure. Additional oxygenation or other means to protect the brain from serious injury may be considered. Seizure activity under general anesthesia may only be recognized by EEG monitoring. The seizure should be adequately controlled with various kinds of anticonvulsant therapy.

Severe coagulopathy should be corrected aggressively perioperatively using fresh frozen or cryoprecipitated plasma, platelet transfusion, and, if fibrinolysis is present, with epsilon-aminocaproic acid. Thromboelastography is very useful in monitoring rapidly changing coagulopathy during transplant surgery. Adequate coagulation is necessary not only to minimize the blood loss, but also to prevent intracranial hemorrhage during the perioperative period.

From the technical point of view, liver transplantation for fulminant hepatic failure is relatively easy. However, in the presence of rapidly deteriorating metabolism and physiology, the operation has to be done smoothly and quickly in order to restore normal liver function as quickly as possible. Immediate good graft function is essential.

\section{RESULTS OF LIVER TRANSPLANTATION FOR FULMINANT HEPATIC FAILURE}

Eight patients with fulminant hepatic failure have received orthotopic liver transplantation under cyclosporine-steroid therapy at the University Health Center of Pittsburgh before July 1985. The clinical data of these eight recipients are summarized in Table 1. All eight patients had had normally functioning livers prior to an acute episode of liver disease and had developed progressive jaundice, coagulopathy, and some degree of hepatic encephalopathy within several weeks.

The cause of massive hepatic necrosis was an adverse reaction to medication in three patients (OT
283 , gold sodium thiomalate; OT 452 , sodium valproate; OT 541, phenytoin sodium), presumed non$A$, non-B virus hepatitis in three patients (OT 341 , OT 373, OT 410 ), B-virus hepatitis in one patient (OT 469), and accidental inhalation of paint fumes in closed space in one patient (OT 283).

Two patients (OT 283, OT 298) had developed severe renal failure and massive gastrointestinal bleeding before transplantation. Two patients (OT 341 , OT 541) had been already in grade IVa encephalopathy, and two others (OT 283, OT 469) had been in deep grade III or occasionally in grade IVa encephalopathy immediately preoperatively.

Four patients (OT 283, OT 298, OT 341, OT 541) died between 2 days and 31 days after transplantation from various causes as listed in Table 1. Both of the patients (OT 341, OT 541) who were definitely in grade IVa encephalopathy at the time of transplant died without any improvement of neurologic function. Both of the patients (OT 283, OT 298) who had had severe renal failure and massive gastrointestinal bleeding at the time of transplant also died.

The other 4 patients (OT 373 , OT 410 , OT 452 , OT 469) are alive between the ninth and seventeenth month postoperatively with good liver function. Neurologic recoveries of surviving patients have been complete without any residual defects in all but one patient. This exceptional patient (OT 452) had regained full consciousness a few days after successful liver replacement, but a myoclonic seizure disorder, which had started two months before iransplantation, has progressed to the point of minimum cerebral cortical function since the second post-transplant month; the patient is now cared for at a nursing home.

One patient (OT 469) with fulminant B-virus hepatitis is alive and well with normal liver functions in the ninth month after transplant. This patient was treated with $100 \mathrm{ml}$ of anti-B virus immunoglobulin during the anhepatic phase of transplant and one month later. He has now antibodies against hepatitis B surface antigen, e-antigen and core antigen.

\section{CONCLUSION}

Although our experience is limited, liver transplantation seems to be an acceptable therapeutic option for fulminant hepatic failure when supportive medical measures appear to be failing. In the presence of grade IV encephalopathy, severe renal failure, and massive gastrointestinal bleeding, liver replacement will probably fail. Because the survival 
TABLE 1. Clinical Data of 8 Patients who Received Orthotopic Liver Transplantation for Fulminant Hepatic Failure

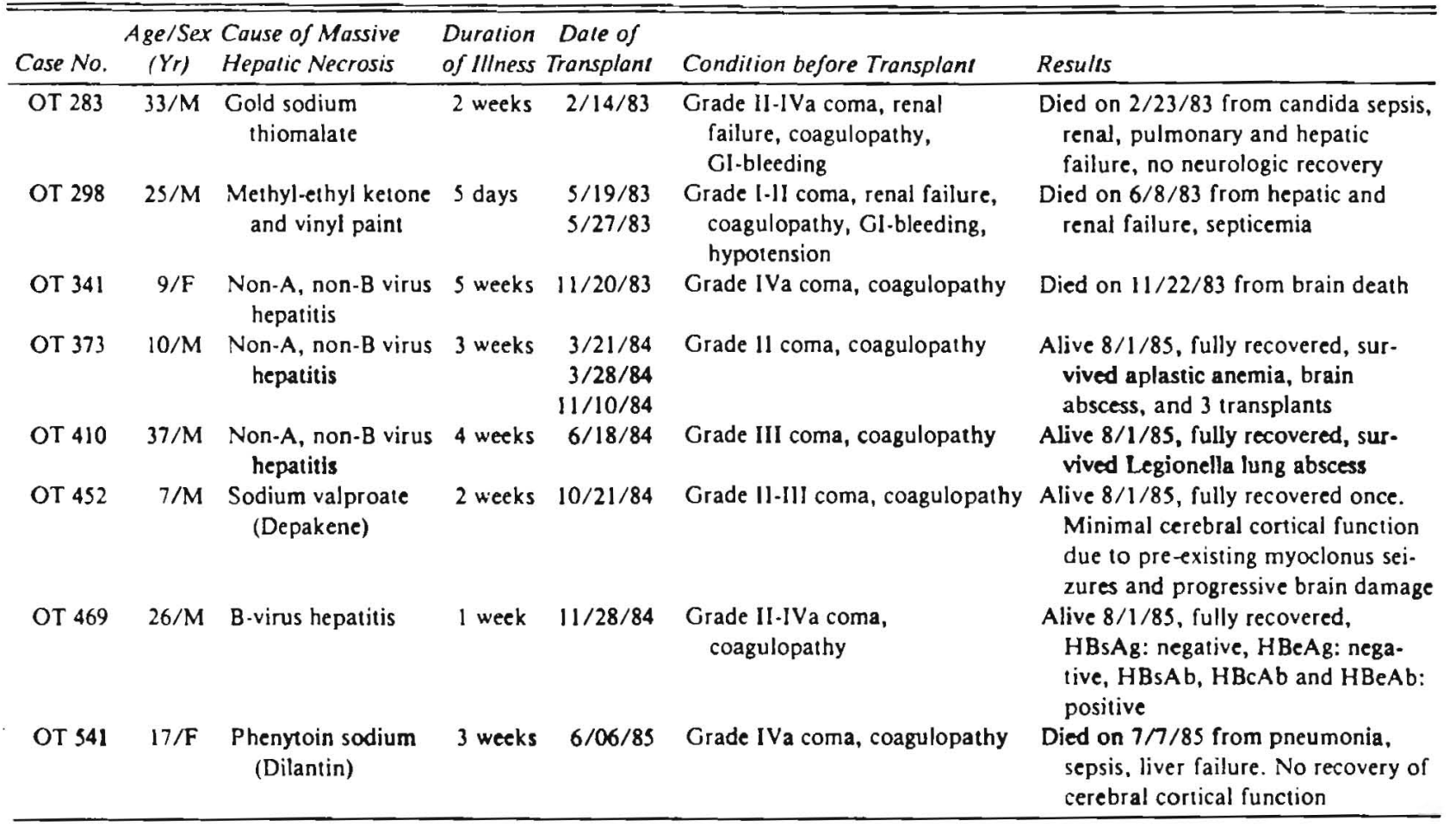

after liver iransplantation has improved in recent years, liver replacement can be justified in somewhat earlier stages of fulminant hepatic failure. Fulminant virus hepatitis does not seem to be an absolute contraindication of liver transplantation, and in one of the recipients, antibodies developed postoperatively while the B-virus surface marker cleared.

Acknowledgment. Supported by Research Grants from the Veterans Administration and Project Grant No. AM-29961 from the National Institutes of Health, Bethesda, Maryland.

\section{REFERENCES}

I. EASL Study Group: Randomized trial of steroid therapy in acute liver failure. Gut 20:20-23, 1979.

2. Ring-Larsen $\mathrm{H}$, Palazzo $\mathrm{V}$ : Renal failure in fulminant hepatic failure and terminal cirrhosis. Gut 22:\$85-592, 1981.

3. Gimson A, Braude S, Mellon P, et al: Earlier charcoal haemoperfusion in fulminant hepatic failure. Lancet 2:691-693, 1982.

4. Williams R, Gimson A: An assessment of orthotopic liver transplantation in acute liver failure. Hepatology 4:22S-24S, 1984.

5. Starzl TE, Iwatsuki S, Van ThieJ DH, et al: Evolution of liver transplantation. Hepatology 2:614-636, 1982.

6. Shaw BW Jr, Martin DJ, Marquez JM, et al: Venous bypass in clinical liver transplantation. Ann Surg 200:524-534, 1984. 